\title{
Spin relaxation in spiral magnetic structures
}

Cite as: Fiz. Nizk. Temp. 47, 94-96 (January 2021); doi: 10.1063/10.0002903

Submitted: 20 November 2020

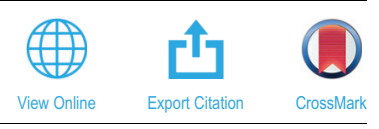

Igor Lyapilin ${ }^{1,2, a)}$

\section{AFFILIATIONS}

${ }^{7}$ Institute of Metal Physics of the Ural Branch of the Russian Academy of Sciences, Ekaterinburg 620108, Russia

${ }^{2}$ Ural Federal University, Department of Theoretical Physics and Applied Mathematics, Ekaterinburg 620002, Russia

\section{a) Author to whom correspondence should be addressed: Lyapilin@imp.uran.ru}

\begin{abstract}
Spin dynamics in spiral magnetic structures has been investigated. It has been shown that the internal spatially dependent magnetic field in such structures produces a new mechanism of spin relaxation.
\end{abstract}

\section{Published under license by AIP Publishing. https://doi.org/10.1063/10.0002903}

\section{INTRODUCTION}

One of the central problems facing spintronics, such as the development of methods of injection, generation, and detection of spin-polarized charge carriers, is to analyze and study the mechanisms of spin relaxation. Spin-orbit interaction (SOI) is well-known to have a significant influence on the mechanisms of electron spin relaxation. SOI can impact on spin degrees of freedom through translational ones.

SOI itself does not cause spin relaxation but in combination with the scattering of the electron momentum leads to its relaxation. For example, Elliott examined the process of electron spin relaxation through the momentum scattering at impurity centers, conditional upon inducing the spin-orbit interaction by lattice ions. ${ }^{2}$ Phonons can also participate in spin relaxation. The nature of spin relaxation involving phonons is the modulation of time-dependent spin-orbit relaxation by lattice oscillations. ${ }^{3}$ As a result, spin relaxation occurs. Thus the Elliott, Yafet mechanisms offer for the spin relaxation frequency $\omega_{s}$ to be proportional to the electron momentum relaxation frequency $\omega_{p}$.

In systems without a center of inversion, a fundamentally different mechanism of spin relaxation can be realized. In such systems, the spin states $\mathrm{t}$ and $\mathrm{X}$ are not degenerate $E_{k \uparrow} \neq E_{k \downarrow}$, but the condition is satisfied $E_{k \uparrow} \neq E_{-k \downarrow}$. The lack of a center of symmetry contributes to splitting the states subjected to SOI. The splitting can be described by introducing an intrinsic magnetic field $\mathbf{B}_{i},(\mathbf{k})$ around which the electron spins precess at a Larmor frequency. The precession of the electron spin in the effective magnetic field together with the electron momentum scattering leads to spin relaxation, and $\omega_{s} \propto \omega_{p}^{-1}$.

\section{SPIRAL MAGNETS}

Here we consider the spin relaxation mechanism in magnetically ordered structures, which are characterized by a spiral arrangement of magnetic moments relative to some crystal axes. ${ }^{5}$ The simplest case of such structures is an antiferromagnetic spiral or a helicoid; they can be found in rare earth metals $(\mathrm{Eu}, \mathrm{Tb}, \mathrm{Dy})$ and some oxide compounds.

The structure of this type can be represented as a sequence of atomic planes perpendicular to the axis of a helicoid. In this case, atoms in each of the planes have ferromagnetically ordered magnetic moments. However, the magnetic moments in neighboring planes are turned at some angle $\theta$ depending on the ratio of exchange interactions. This is because of the coexistence of positive exchange interaction between the nearest atomic neighbors and negative exchange interaction between the neighbors following the closest ones. The components of the magnetic moments of atoms oscillate in the plane of the magnetic layer $S_{x}=S_{0 x} \sin k z, S_{y}=S_{0 y} \cos k z$. If, in this case, $S^{z} \neq 0$, we have a ferromagnetic spiral with a resulting moment. If also oscillates $S^{z}$ by a harmonic law, a complex magnetic structure emerges.

In such structures, the exchange interaction between zone charge carriers and localized moments is described by the wellknown expression $H_{\mathrm{ex}}=-J \sum_{i} \mathbf{s} \cdot \mathbf{S}$ where $\mathrm{J}$ is the exchange constant. In the mean-field approximation, this interaction can be represented as $H_{\mathrm{ex}}=-\mathbf{m}(\mathbf{r}) \mathbf{H}_{\mathrm{ef}}(\mathbf{r})$, where $\mathbf{m}(\mathbf{r})=g \mu_{B} \mathrm{~S}(\mathbf{r}), \mathrm{g}$ is the spectroscopic splitting factor, $\mu_{\mathrm{B}}$ is the Bohr magneton, and $\mathbf{H}_{\mathrm{ef}}(\mathbf{r})$ is the internal effective magnetic field.

The spatial variation of the internal field in spiral magnetic structures should affect the spin dynamics of conduction electrons. Indeed, the spin of an electron in a state with quasi-momentum $\mathbf{k}$ precesses in the effective magnetic field $\mathbf{H}_{\mathrm{ef}}(\mathbf{r})$ only for a time of the order of the elastic scattering time $\tau_{p}$. After scattering, the electron goes into a state $\left(\mathbf{k}^{\prime}\right)$ where the effective magnetic field has a different direction. Consequently, the evolution of the spin dynamics under such conditions turns out to be associated with the electron momentum relaxation. 
Let us look at a simple spiral where the effective magnetic field rotates in a plane $(x y)$, where

$$
\mathbf{H}_{\mathrm{ef}}=\left(H_{0} \cos (Q z), H_{0} \sin (Q z), 0\right),
$$

$\mathrm{Q}=2 \pi / \Lambda, \Lambda$ is the period of the spiral structure. To gain insight into the spin dynamics, we estimate the spin relaxation mechanism realized under above conditions. The system at hand is assumed to expose to an electric field directed along the axis $z\left[\mathbf{E}=\left(0,0, \mathrm{E}_{0}\right)\right]$.

The Hamiltonian of the system can be written in the form

$$
H=H_{k}+H_{s}+H_{e E}+H_{v}+H_{e v},
$$

where $H_{k}=\sum p_{i}^{2} / 2 m, \quad H_{s}=-g \mu_{B} \sum \mathbf{s}_{i} \mathbf{H}_{\mathrm{ef}}$, are the operators of the kinetic and spin energy of electrons interacting with the lattice $H_{e v}$ and the external electric field $H_{e E}=-e \sum \mathbf{E r}_{i} \cdot H_{v}$ is the Hamiltonian of the lattice. Taking into account the expression $\mathrm{H}_{\mathrm{ef}}$ we get

$$
H_{s}=-\hbar \omega_{s f} \sum_{j}\left(s_{j}^{+} \mathrm{e}^{i Q z_{j}}+s_{j}^{-} \mathrm{e}^{-i Q z_{j}}\right),
$$

where $\omega_{s f}=g e H_{0} / 2 m_{0} c$ is the electron precession frequency in the effective field, $\mathrm{s}^{ \pm}=s_{x} \pm$ is $s_{y}$.

\section{MACROSCOPIC EQUATIONS}

The spin dynamics is determined by macroscopic equations of motion for the spin subsystem of electrons. The macroscopic equations of motion can be deduced by averaging microscopic equations of motion over the nonequilibrium statistical operator (NSO) $\rho(t)$. To analyze the kinetics of the spin effects, we employ a scheme developed in the NSO method ${ }^{6}$ applied to the case of a small deviation of the system from the equilibrium Gibbs distribution $\rho_{0}=\exp$ $\left\{-S_{0}\right\}$. The entropy $S_{0}$ of the equilibrium system with the Hamiltonian $\mathrm{H}$ can be written as

$$
S_{0}=\Phi_{0}+\beta\left(H_{k}+H_{s}-\mu N\right)+\beta\left(H_{v}+H_{e v}\right),
$$

where $\beta^{-1}=\mathrm{T}$ is the equilibrium temperature of the system. $\mathrm{N}$ is the operator of the particle number, $\mu$ is the chemical potential of electrons.

The non-equilibrium state of the system there corresponds the entropy operator

$$
\begin{aligned}
S(t) & =\Phi(t)+\left\{\beta\left(H_{k}-\mu(t) N\right)+\beta_{s}(t) H_{s}+\beta\left(H_{v}+H_{e v}\right)\right\} \\
& =S_{0}+\delta S(t) .
\end{aligned}
$$

$\Phi(t)$ is the Massieu-Planck functional. $\beta_{s}$ is the value of the inverse temperature of the spin subsystem.

In the linear approximation the deviation from the equilibrium, the NSO (or the density matrix) $\rho(t)$ can be written as follows

$$
\rho(t)=\rho_{q}(t)+\int_{-\infty}^{0} d t_{1} \mathrm{e}^{\varepsilon t_{1}} \int_{0}^{1} d \tau \rho_{0}^{\tau} S\left(t+t_{1}, t_{1}\right) \rho_{0}^{-\tau} \rho_{0} .
$$

Here $\rho_{q}(t)=\exp \{-\mathrm{S}(\mathrm{t})\}$ is the quasi-equilibrium statistical operator, $\mathrm{S}(\mathrm{t})$ being the entropy production operator:

$$
\dot{S}(t)=\delta \dot{S}(t)=\frac{\partial S(t)}{\partial t}+\frac{1}{i \hbar}[S(t), H] .
$$

$\dot{S}\left(t+t^{\prime}, t^{\prime}\right)=U_{t} \dot{S}\left(t+t^{\prime}, 0\right) U_{-t}, U_{t}$ is the evolution operator with hamiltonian $H$.

A further algorithm for constructing the operator $\rho(t)$ comes to finding the entropy production operator. Commuting the operators $s^{z}, p^{z}$ with the Hamiltonian (1), we find the microscopic equations of motion for the longitudinal components of the spin and momentum of electrons. We have

$$
\dot{s}^{z}=i \omega_{s f}\left\{s^{+} \mathrm{e}^{i Q z}-s^{-} \mathrm{e}^{-i Q z}\right\}+\dot{s}_{e v}^{z},
$$

$$
\dot{p}^{z}=-e E N+i Q \hbar \omega_{s f}\left\{s^{+} \mathrm{e}^{i Q z}-s^{-} \mathrm{e}^{-i Q z}\right\}+\dot{p}_{e v}^{z} .
$$

Here $\mathrm{A}=(i \hbar)^{-1}[A, H], A_{i j}=(i \hbar)^{-1}\left[A, H_{i j}\right]$.

Using the operator equations of motion we can construct an expression for the entropy production operator $\dot{S}(t)$ in a linear approximation over $H_{e v}$ interaction

$$
\begin{gathered}
\rho=\rho_{q}(t, 0)-\frac{1}{i \hbar} \int_{-\infty}^{0} d t^{\prime} e^{\varepsilon t^{\prime}}\left[\rho_{q}(t, 0), U_{t}^{0} H_{e v} U_{-t}^{0}\right], \\
\rho_{q}(t, 0)=-S(t, 0), \quad U_{t}^{0}=U_{t}\left(H_{e v}=0\right) .
\end{gathered}
$$

Averaging the microscopic equations of motion over the operator $\rho$ $(t)$, we obtain $\left\langle A_{i}\right\rangle^{t}=\operatorname{Sp}\left\{A_{i} \rho(t)\right\}$. As a result, we come to the following system of macroscopic equations.

$$
\left\langle\dot{s}^{z}\right\rangle^{t}=i \omega_{s f}\left\{\left\langle s^{+} e^{i Q z}\right\rangle^{t}-\left\langle s^{-} e^{-i Q z}\right\rangle^{t}\right\}+\left\langle\dot{s}_{e v}^{z}\right\rangle^{t}
$$

and

$$
\left\langle\dot{p}^{z}\right\rangle^{t}=-e E n_{0}+i Q \hbar \omega\left\{\left\langle s^{+} e^{i Q z}\right\rangle^{t}-\left\langle s^{-} e^{-i Q z}\right\rangle^{t}\right\}+\left\langle\dot{p}_{e v}^{z}\right\rangle^{t},
$$

where $\langle N\rangle^{t}=n$ is the electron concentration.

The collisional summands in the balance Eqs. (7) and (8) can be represented as follows: ${ }^{6,7}$

$$
\left\langle\dot{s}_{e v}^{z}\right\rangle^{t} \sim\left(s^{z}, s^{z}\right)_{0} \omega_{s},\left\langle\dot{p}_{e v}^{z}\right\rangle^{t} \sim\left(p^{z}, p^{z}\right)_{0} \omega_{p}
$$

Here $\omega_{s}$ is the relaxation frequency of the longitudinal spin components, and $\omega_{p}$ is the momentum relaxation frequency; both latter are calculated in the Born approximation over the scatter- 
electron interaction.

$$
\begin{gathered}
\omega_{s}=\frac{g \mu_{B}}{\chi} \int_{-\infty}^{0} d t \mathrm{e}^{\varepsilon t}\left(\dot{s}_{e v}^{z}, \dot{s}_{e v}^{z}(t)\right)_{0}, \\
\omega_{p}=\frac{1}{m n T} \int_{-\infty}^{0} d t \mathrm{e}^{\varepsilon t}\left(\dot{p}_{e v}^{z}, \dot{p}_{e v}^{z}(t)\right)_{0}, \quad \varepsilon \rightarrow+0 .
\end{gathered}
$$

Here $\chi$ is the paramagnetic susceptibility of an electron gas

$$
\chi=\frac{\left(g \mu_{B}\right)^{2}}{2 T} \cdot\left(s^{+}, s^{-}\right)_{0}=\frac{g \mu_{B}}{H_{0}} \cdot\left\langle s^{z}\right\rangle_{0},
$$

$T=k_{B} T$ is the temperature in energy units. $(A, B)_{0}$ is the correlation function

$$
(A, B)_{0}=\int_{0}^{1} d \tau S p\left\{A \rho_{0}^{\tau} \Delta B \rho_{0}^{1-\tau}\right\},
$$

where $\Delta A=A-\langle A\rangle_{0},\langle\cdots\rangle_{0}=\operatorname{Sp}\left\{\cdots \rho_{0}\right\}, \rho_{0}$ is the equilibrium Gibbs distribution.

Taking a stationary case into account, we finally obtain the expression for the spin relaxation frequency in a helicoidal magnet:

$$
\omega_{s} \propto \frac{m n T}{\hbar Q\left(s^{z}, s^{z}\right)_{0}} \cdot \omega_{p}
$$

\section{CONCLUSIONS}

Thus, in spiral magnets as well as in the Elliott, Yafet mechanisms, the spin relaxation is related to the electron momentum relaxation and $\omega_{s} \propto \omega_{p}$. However, unlike the Elliott, Yafet mechanisms, the spin relaxation mechanism in spiral magnetic structures is due to the presence of the internal spatially-dependent magnetic field in them. The evolution of the spin dynamics is determined by both the period of the magnetic structure and the momentum relaxation frequency.

\section{ACKNOWLEDGMENTS}

The research was carried out within the state assignment of Ministry of Science and Higher Education of the Russian Federation (theme "Spin” No. AAAA-A18-118020290104-2), supported in part by RFBR (Project No. 19-02-00038/19).

\section{REFERENCES}

${ }^{\mathbf{1}}$ E. I. Rashba, Physica E 20, 189 (2004).

${ }^{2}$ R. J. Elliott, Phys. Rev. 96, 266 (1954).

${ }^{3}$ Y. Yafet, Solid State Phys., edited by F. Seitz and D. Turnbull (New York Academic, 1963), Vol. 14.

${ }^{4}$ M. I. Dyakonov and V. I. Perel, Sov. Phys. Solid State 13, 3023 (1972).

${ }^{5}$ Yu. A. Izyumov, Sov. Phys. Usp. 27, 845 (1984).

${ }^{6}$ I. I. Lyapilin, M. S. Okorokov, and V. V. Ustinov, Phys. Rev B 91, 195309 (2015).

${ }^{7}$ H. M. Bikkin and I. I. Lyapilin, Non Equlibrium Thermodynamics and Physical Kinetics (Walter de Gruyter GmbH, Berlin, 2015), p. 359.

Translated by AIP Author Services 